\title{
TTR
}

Traduction, terminologie, re?daction

\section{Louise-L. Larivière. Pourquoi en finir avec la féminisation linguistique ou À la recherche des mots perdus. Montréal, Éditions du Boréal, 2000, 149 p.}

\section{Claude Tatilon}

Volume 14, numéro 2, 2e semestre 2001

Antoine Berman aujourd'hui

Antoine Berman for our time

URI : https://id.erudit.org/iderudit/000577ar

DOI : https://doi.org/10.7202/000577ar

Aller au sommaire du numéro

\section{Éditeur(s)}

Association canadienne de traductologie

ISSN

0835-8443 (imprimé)

1708-2188 (numérique)

Découvrir la revue

Citer ce compte rendu

Tatilon, C. (2001). Compte rendu de [Louise-L. Larivière. Pourquoi en finir avec la féminisation linguistique ou À la recherche des mots perdus. Montréal, Éditions du Boréal, 2000, 149 p.] TTR, 14(2), 225-232.

https://doi.org/10.7202/000577ar d'utilisation que vous pouvez consulter en ligne.

https://apropos.erudit.org/fr/usagers/politique-dutilisation/ 


\section{COMPTES RENDUS}

\section{Louise-L. Larivière. Pourquoi en finir avec la féminisation linguistique ou À la recherche des mots perdus. Montréal, Éditions du Boréal, 2000, 149 p.}

Le titre est ambigu : il « peut, en effet, s'interpréter différemment selon que l'on est pour ou contre la féminisation ». L'auteure est pour, évidemment, voulant « en finir avec toutes les faussetés qui circulent autour de cette notion : faussetés de croire que la féminisation est un phénomène nouveau des vingt dernières années, qu'elle est née du cerveau enflammé des féministes québécoises, qu'elle ne se manifeste qu'au Québec et qu'elle ne vaut que pour la langue française » (p. 13).

Habile plaidoyer en faveur de la féminisation, l'ouvrage n'a qu' « un seul but : contrer le sexisme dans la langue » (p. 16). Il s'impose par sa logique et son bon sens, autant que par son « style incisif et mordant, teinté parfois d'humour et de sarcasme " - la quatrième de couverture ne ment pas.

Le propos de l'étude est d'examiner « la raison d'être de la féminisation linguistique ainsi que des phénomènes qui font obstacle soit à la visibilité des femmes (masculinisation de la langue), soit à l'égalité entre les femmes et les hommes (marginalisation du féminin), et ce, en décrivant les formes d'opposition à la féminisation, formes marquées, bien souvent, du sceau de l'ignorance, de la bêtise et de la mauvaise foi. Le comment de la question est traité dans un livre numérique aux Éditions www.00h00.com (Comment en finir avec la féminisation linguistique ou Les mots pour la dire) » (p. 17). Ce dernier ouvrage, disponible depuis peu, ne fait pas partie du présent compte rendu.

Les attaques contre la féminisation sont ici remarquablement synthétisées - en huit points (pp. 89-118) qui méritent d'être énumérés :

1. « La femme de... » : une expression malheureuse qui a la vie dure. 
2. «Ce nom est déjà pris »: sophisme en vertu duquel l'homonymie serait à proscrire pour les nouveaux noms féminins. Une manœuvre, une critique ne seraient alors pas acceptables pour dénommer une femme, en dépit du secrétaire, du patron, du guide et de bien d'autres noms attribués aux hommes.

3. Certains suffixes féminins sont péjoratifs, -euse en particulier, comme dans niaiseuse, peureuse, " téteuse " (au sens québécois de lambine). Mais ici la péjoration provient de la base lexicale (niais, peur, téter) et non du suffixe, du reste bien toléré dans de nombreux cas comme chanteuse, danseuse, coiffeuse...

4. Écrivaine, quelle horreur! Le nom rime avec vaine, déclare-t-on sans se soucier le moins du monde de la rime d'écrivain! Les noms auteure, professeure seraient laids ou barbares ; agente de police imprononçable, en dépit de parente, régente... Reconnaissons-le, l'argument pseudoesthétique ne fait pas très sérieux.

5. Une ministre, une juge, « ce n'est pas dans le dictionnaire ». Ces deux noms ont naguère fait couler beaucoup d'encre du côté de l'Académie française. Bien inutilement car ils sont déjà dans presque toutes les bouches.

6. Annonceuse, meneuse, entraîneuse, " ce n'est pas du français ». Mais, curieusement, l'adjectif substantivé emmerdeuse n'est, lui, jamais contesté.

7. Le dénigrement des noms féminins passe aussi, souvent, par de mauvaises plaisanteries dignes « de jeunes collégiens ", dont sont très friands certains «Immortels» : Jean Dutourd proposant majordame et enseignette de vaisselle pour faire pendant à majordome et à enseigne de vaisseau; Marc Fumaroli recommandant la légalisation des noms en -esse (notairesse, mairesse, doctoresse) parce qu'ils riment avec fesse ! 
8. Un titre prestigieux au féminin est péremptoirement déclaré discriminatoire (voir le grand débat de 1998 autour de la ministre).

Ces trente pages constituent une synthèse quasi exhaustive des coups bas couramment portés à la féminisation en français. L'argumentation de l'auteure est serrée, ses explications, convaincantes :

« [U]n titre féminin n'est pas menaçant dans la mesure où il se limite à désigner des fonctions traditionnelles dévolues aux femmes. S'il devient menaçant, on lui fera jouer une autre fonction ou bien on lui préfèrera le titre masculin. Il ne viendra pas à l'idée des adversaires de la féminisation d'accorder au genre féminin le même statut et la même valeur qu'au genre masculin » (p. 85).

" Curieusement, comme on l'a vu précédemment, on ne s'opposera pas à la distinction en genre pour ce qui est des fonctions traditionnelles : un instituteur/une institutrice, pas plus que l'on ne s'opposera à la création d'une appellation masculine lorsque les hommes accèdent à des fonctions traditionnellement féminines (ex. un maïeuticien). Pourquoi alors s'oppose-t-on à la création d'une appellation féminine lorsque les femmes accèdent à des fonctions traditionnellement masculines : ingénieure, doyenne, rectrice, etc ? Et pourquoi trouver bizarres, laides ou ridicules ces appellations ? » (p. 88)

" Aussi s'agit-il, tout simplement, de "se faire l'oreille" à ces nouvelles appellations pour ne plus les trouver bizarres. En fait, on a affaire à un phénomène circulaire : moins on utilisera ces termes, plus ils nous paraîtront inhabituels, alors que leur usage répété fera en sorte qu'on ne remarquera même plus leur caractère de nouveauté » (p. 99).

Les trois substantifs avancés plus haut - ignorance, bêtise, mauvaise foi - constituent bien, en l'occurrence, des mots justes. L'ouvrage, qui fait 
mouche à tout coup, est à lire et à faire lire. En priorité aux élèves de nos écoles de traduction avec lesquels il est indispensable d'ouvrir le débat sur une question épineuse, « hautement émotive » (p. 12), qu'ils auront à affronter tout au long de leur carrière.

La première préoccupation de l'auteure étant « de rendre le propos accessible à toute personne qui s'intéresse à cette question ", force m'est de signaler une faiblesse de l'ouvrage : il n'est pas exempt d'un certain flou terminologique. Flou qui, fort malencontreusement, affecte la notion même de genre, située en plein cœur du débat. Considérons le titre du troisième chapitre : "Le masculin : un genre neutre ». Le jeu de mots qu'il renferme repose sur la collision de plusieurs sens. Au fil de l'ouvrage, le substantif genre voit son sens osciller du domaine de la grammaire à celui des sciences naturelles. On lit, page $59:$ : en anglais (américain à tout le moins), on a aboli les titres ayant une marque de genre au profit d'un genre indifférencié ou commun (common gender) pour les titres professionnels : ex. Airplane-flight attendant au lieu de air steward et air stewardess ». Ici, genre, comme l'anglais gender, signifie « sexe " ; il en va de même plus bas, à la page 102, où l'auteure fait mention « des titres professionnels indifférenciés pour les deux genres ». Et, à la page 107, où elle écrit : « On pourrait, toutefois, dégenriser certaines expressions, comme réduire "une chasse à l'homme" à "une chasse" (tout court). ", le néologisme dégenriser veut dire faire disparaître tout substantif désignant le " genre humain » (comme on dit toujours dans les sciences naturelles). Alors, genre grammatical, sexe ou humanité ? Les lecteurs ont besoin de savoir, à chaque moment de leur lecture, à quelle notion ils ont affaire.

Le genre grammatical, qui est un phénomène morphologique portant excusivement sur les noms et leurs déterminants (on ne le rencontre pas dans toutes les langues), ne correspond pas à un monème (unité minimale de sens) et n'a donc pas de signifié. Comme le précise le linguiste André Martinet, « [i]l y a en français, et c'est tout autre chose, un monème de sexe féminin, manifeste dans le -esse de princesse ou d'ânesse. " (Grammaire fonctionnelle du français. Crédif/Didier, Paris, 1979, p. 12) Il faut aussi parler d'un monème beaucoup plus rare - de sexe masculin, qu'on peut trouver dans le - ard de canard et dans le -et de mulet (ou, en anglais, dans le -er de widower). 
Il est bon aussi de signaler que le sexe linguistique connaît trois valeurs — « mâle », comme avec garçon ou bélier ; « femelle », comme avec fille ou brebis ; "sexué » sans plus de précision, comme avec personne ou mouton - et qu'il peut se manifester de trois façons différentes en français : par un monème prenant la forme d'un suffixe (princ-esse), par un trait de sens lexical (c'est le cas avec les lexèmes sœur, père, tante) et par le seul genre grammatical, qu'on peut alors considérer comme sémantisé, dans le cas des noms épicènes (pianiste, alpiniste, archiviste). Il est évident, de surcroît, que les noms porteurs d'une information sexuelle sont généralement (mais pas systématiquement : un mannequin, une sentinelle) dotés du genre grammatical correspondant.

La séparation, en langue, du genre grammatical et du signifié « sexe » n'est pas chose facile, ce qui explique la confusion qui règne dans l'esprit du locuteur moyen quant au genre grammatical. Il est donc impératif, dans les écrits scientifiques et pédagogiques, de multiplier les précautions terminologiques pour bien séparer le genre grammatical, phénomène morphologique, du signifié « sexe ", monème ou simple trait de sens.

Quant au neutre, il n'existe pas en français en tant que genre grammatical. L'auteure le dit clairement (p. 51) et réprouve le rôle neutralisant assumé par le masculin. Dans le chapitre 3 (« Le masculin : un genre neutre »), neutre désigne le pouvoir sémantique qu'ont les noms masculins de neutraliser - au pluriel (les habitants, les étudiants, les voyageurs, les touristes) et même, pour certains d'entre eux, au singulier (un membre, un personnage, un protagoniste, le conjoint) - la caractérisation sexuelle. Mais, ici encore, on perd souvent le fil de la pensée, hésitant entre les deux acceptions : « sexe indifférencié » ou genre grammatical ? Et le rapprochement avec le chapitre qui précède (« Le masculin : un genre générique ») ne facilite pas la compréhension: existe-t-il une différence réelle entre le masculin générique et le masculin neutralisant ? Et, si l'on tient à une distinction aussi ténue, conserve-t-elle un quelconque intérêt pour l'analyse du phénomène de la féminisation?

Un terme, c'est une perche qui doit permettre de sauter pardessus les nébulosités de la pensée et d'aller plus loin dans le raisonnement. Pour être scientifique, un raisonnement se doit d'être rigoureux avec la terminologie qu'il utilise, ce qui signifie bien choisir ses termes, clairement les définir et les affecter d'une valeur 
permanente. C'est à cette seule condition qu'il devient linguistiquement communicable.

Je ne puis me résoudre à clore la discussion sans signaler une erreur qui me tient particulièrement à cœur. Après avoir précisé que « [1]a féminisation doit, d'abord, se faire en tenant compte de la réalité propre d'une langue » (p. 31) et qu' « il ne s'agit pas de "copier" les mesures, toutes intéressantes qu'elles soient, prises dans d'autres langues dont les structures diffèrent de celles du français »(p. 32), Louise-L. Larivière s'en prend à l'un de mes articles, me faisant dire par un flagrant contresens - que « la question de la féminisation n'est pas un phénomène français »!?! Me donnant du « professeur français » (ce qui n'est pas une insulte mais une inexactitude : né en France, je suis devenu citoyen canadien voilà déjà longtemps et j'enseigne en Ontario depuis... 34 ans seulement), elle me place, aux pages 102-103 de son livre, "en sandwich » entre l'ex-garde des Sceaux français, Jacques Toubon, et les académiciens Marc Fumaroli et Maurice Druon - position que je trouverais plus flatteuse qu'inconfortable s'agissant d'un tout autre sujet que la féminisation. Et j'avoue que cet ostracisme me laisse perplexe, ayant écrit, dans l'article cité (à l'évidence trop vite lu), que « des différences importantes séparent le français de l'anglais » et précisé qu' " on doit toujours les garder à l'esprit, surtout lorsqu'on trouve séduisantes certaines solutions prônées par les féministes américaines ou canadiennes-anglaises pour épurer le discours : leurs solutions sont rarement les nôtres. La langue française (à cause de son genre grammatical incontournable et de sa dérivation omniprésente) favorise la féminisation ; la langue anglaise (à cause de l'absence de genres et d'accord à l'intérieur du syntagme nominal, du grand nombre de noms épicènes - agent, author, clerk, director, novelist, patron, student... - et de la haute fréquence de l'élément man dans les composés - businessman, chairman, congressman, layman, mailman, policeman ; manhole, manhood, mankind, manpower...) — connaît à peine la féminisation (empruntée le plus souvent aux langues romanes et ressentie comme superflue, ridicule, voire désobligeante : bachelorette, doctoress, majorette, poetess, stewardess, suffragette - au lieu de suffragist) et favorise donc très nettement la neutralisation $»(\mathrm{p} .142)$. Louise-L. Larivière dit en gros la même chose (pp. 13, 31-32, 102-103). À une exception près : elle ne dit pas que, stricto sensu, l'anglais n'est plus une langue à genres. L'eût-elle dit, ses commentaires sur cette langue et sur sa comparaison avec la nôtre eussent été beaucoup plus pertinents. « [L]a 
féminisation [...] est un phénomène incontournable et irréversible qui est là pour demeurer tant que les langues auront des genres ", écrit-elle, fort justement, en conclusion de son ouvrage (p. 120). En conséquence, appliqué à l'anglais, le terme féminisation est un faux sens. À moins de vouloir l'affubler d'une acception supplémentaire qui ne manquerait pas d'en ruiner l'univocité.

Pour en terminer avec la différence structurale profonde qui sépare le français, langue à deux genres grammaticaux, de l'anglais, langue qui a renoncé aux siens depuis longtemps, "il n'est peut-être pas indifférent de constater, dit André Martinet, quelle faible valeur informative a l'appareil morphologique très lourd, qu'entraîne en français l'existence du genre ». Et d'expliquer :

"On n'hésite pas à identifier il et elle comme les équivalents des pronoms anglais he et she qui, eux, se réfèrent directement à une personne et non à un mot, dans une langue qui a su se débarrasser des genres. Les adjectifs possessifs his et her rappellent directement le sexe du possesseur, alors qu'en français les correspondants de $i l$ et elle sont indistinctement son et $s a$ qui s'accordent en genre avec le substantif suivant désignant le possédé, ce qui implique qu'ils n'apportent aucune information. Ceci permet d'employer son devant un mot de genre féminin lorsque le $s a$ qu'on attendrait précéderait une voyelle initiale, dans son équipe, par exemple. » ("Genre et sexe ». La linguistique, vol. 35, fasc. 2/1999, pp. 5-9)

J'avoue que, devant l'interprétation faussée que Louise-L. Larivière donne à mon article, quelques doutes m'ont traversé l'esprit quant au sérieux dont elle avait pu faire preuve en consultant les nombreuses études consignées dans sa bibliographie. Mais trève de mesquinerie ! Considérons avant tout la richesse et la pertinence de cette bibliographie, à laquelle je me permettrai d'ajouter trois titres : Françoise Héritier, Masculin/Féminin. La pensée de la différence (Éditions Odile Jacob, Paris, 1996) ; Paul Garde, « Les mots vieux garçons. Réponse à Marc Fumaroli » (Le Monde, mardi 11 août 1998); Claude Tatilon, « Un genre bien à elles » (La linguistique, vol. 34, fasc. 1/1998, pp. 107-122) .

Les objections que je viens de formuler (dans un esprit constructif) ne m'empêcheront pas de répéter in fine que Pourquoi en finir avec la féminisation linguistique ou À la recherche des mots perdus est un livre dont je recommande vivement la lecture à 
quiconque se préoccupe d'équité linguistique. Livre que je referme sur le pressant désir de lire le Comment à la suite du Pourquoi.

\title{
Claude Tatilon Collège Glendon, Université York
}

\begin{abstract}
Yves Gambier and Henrik Gottlieb (eds.). (Multi) Media Translation: Concepts, Practices, and Research, John Benjamins Publishing Company, 2001, 298 p.
\end{abstract}

(Multi) Media Translation is a collection of twenty-six articles that addresses how the rapid changes in technology and the globalization of communication networks have added new domains to the field of translation and are challenging the translator's role. The contributions are the selected works from two recently organized events: the Misano Seminar on Multimedia \& Translation (September 26-27, 1997); and the Berlin conference on Quality and Standards in Audiovisual Language Transfer (October 15-16, 1998).

The twenty-six articles, together with an introduction and an epilogue, are divided into three sections: "Concepts", "Policies and Practices" and "Empirical Research". Some topics tend to overlap, but each article offers a fascinating look at the various aspects of multimedia translation.

Part One, titled "Concepts", has seven articles in total. This section ponders the relationship between media and multimedia, verbal and multimedia translation, the links between text, images and sounds, the new models of communication, the new elements and parameters of translation for the cinema, video, television, CD-ROM, the Web, etc. Emphasis is placed on the need for Translation Studies to collaborate across many disciplines such as Communication Studies, Media and Film Studies, Cultural Studies, Information Sciences and Computer Sciences. Several papers, notably Patrick Cattrysse's "Multimedia \& Translation: Methodological Considerations" and Aline Remael's "Some Thoughts on the Study of Multimodal and Multimedia Translation", aim to define and conceptualise the commonly employed term multimedia. They examine the role of language and picture, and 